\title{
THE EFFECT OF EPINEPHRINE (USP), $l$-EPINEPHRINE, AND $l$-NOREPINEPH- RINE ON GLOMERULAR FILTRATION RATE, RENAL PLASMA FLOW, AND THE URINARY EXCRETION OF SODIUM, POTASSIUM, AND WATER IN NORMAL MAN 1, 2
}

\author{
By CHEVES McC. SMYTHE, ${ }^{3}$ JAMES F. NICKEL,4 AND STANLEY E. BRADLEY \\ (From the Department of Medicine, Columbia University College of Physicians and Surgeons, \\ and the Presbyterian Hospital in the City of New York)
}

(Submitted for publication December 3, 1951; accepted March 24, 1952)

The adrenal medulla apparently elaborates at least two highly active pressor principles, $l$-epinephrine and $l$-norepinephrine, that may profoundly affect the renal circulation in man. In physiologic dosage both substances evoke an intrarenal vasoconstriction which tends to reduce renal blood flow in the face of an elevated arterial pressure without significant alteration in glomerular filtration rate (1-3). Associated changes in urine formation might be predicted but have not as yet been clearly defined. In animals, $l$-epinephrine seems to give rise to a water diuresis and augmented excretion of chloride (4-8), whereas in man both agents may diminish urine flow $(1,2)$. A study of the effect of pure $l$-epinephrine and $l$-norepinephrine upon renal hemodynamics, and excretion of water and electrolytes in normal human subjects was undertaken in order to clarify this problem. Since most of the earlier work is based upon the use of adrenal extracts which have been found to contain both pressor amines $(9,10)$, the effect of epinephrine (USP) was also examined in order to ascertain whether its action is specific or predominantly that of one of its ingredients.

\section{METHODS}

The subjects of this study were 17 convalescent patients (seven male, ten female) from the wards of the Presbyterian Hospital in the City of New York. All were free of clinically demonstrable cardiac and renal disease. They received a well-balanced hospital diet containing approximately 10 grams of sodium chloride each day.

1 This work has been aided by a grant from the American Heart Association.

2 These data were presented in detail before the International Congress of Clinical Pathology, London, England, on July 19, 1951 (33).

${ }^{3}$ Research Fellow of the American College of Physicians.

Fellow of the New York Heart Association.
The effects of $l$-norepinephrine 5 were determined on 12 occasions, and of $l$-epinephrine ${ }^{6}$ and epinephrine (USP) three times each. Glomerular filtration was measured as the inulin clearance, and renal plasma flow as the sodium $p$-aminohippurate clearance. ${ }^{7}$ The renal clearances of sodium and potassium were determined simultaneously. The blood pressure was measured sphygmomanometrically, and mean pressure calculated after the method of Böger and Wezler (11) for use in computation of renal vascular resistance. The methods employed in the experimental procedures and chemical analyses have been described at length elsewhere $(12,13)$. All solutions were made up in $5 \%$ dextrose and distilled water. Three successive resting control clearance values were determined at ten-minute intervals prior to intravenous administration of an infusion of the pressor agent under study (4 to $8 \mu \mathrm{g}$./ ml. of $5 \%$ dextrose in distilled water) at a rate which was maintained constant at about $15 \mu \mathrm{g} . / \mathrm{min}$. in most individuals. In one very susceptible subject the rate of infusion ( $l$-epinephrine) could not be raised above $2 \mu \mathrm{g} . /$ min. without discomfort, and in another $46 \mu \mathrm{g} . / \mathrm{min}$. of $l$-norepinephrine was required to produce the desired effect. An effort was made to maintain the mean arterial tension between 25 and $50 \mathrm{~mm}$. $\mathrm{Hg}$ above the control without undue subjective reaction. These precautions eliminated the possible role of fright or discomfort in producing the observed effects. The urine voided during the period of equilibration of the arterial pressure at a constant level was discarded and three successive determinations were then made over an interval of from 30 to 45 minutes. In ten instances, two more clearance values were obtained after the blood pressure had returned to control levels and after a 15-minute discard period to permit equilibration.

\section{RESULTS}

Systemic circulatory adjustments and subjective response. The systemic hemodynamic and

5 Supplied through the courtesy of the Medical Research Department, Winthrop-Stearns, Inc., as "Levophed."

- We are indebted to Dr. Maurice Rapport for a supply of pure $l$-epinephrine.

${ }^{7}$ We are indebted to Dr. W. Boger and the Medical Research Division of Sharp and Dohme, Inc., Philadelphia, $\mathrm{Pa}$., for a generous supply of sodium $p$-aminohippurate. 


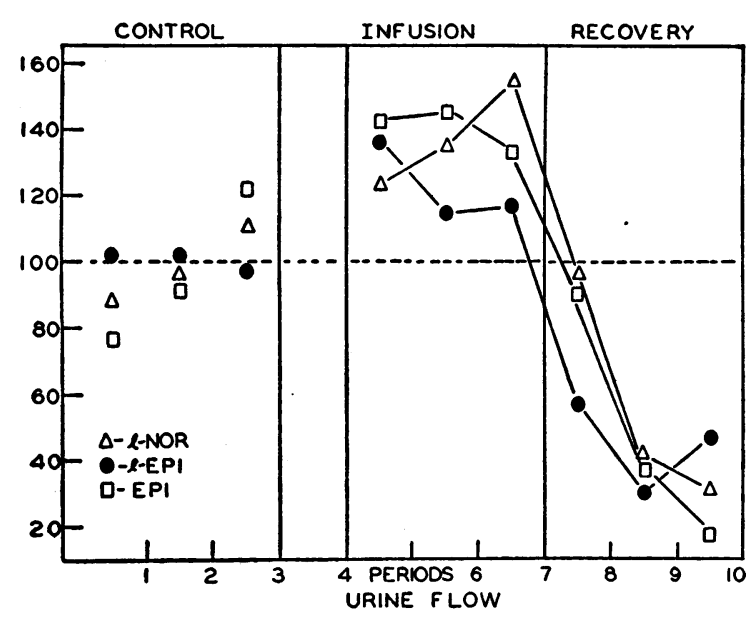

Fig. 1. The EFfect of Epinephirine (USP), l-EpiNEPHRINE AND $l$-NOREPINEPHRINE ON URINE FLOW

The values for urine flow during each "period" (usually ten to 15 minutes in length) have been averaged and expressed here as the percentage of the pooled and averaged control figures. An infusion of epinephrine (USP) (open squares), l-epinephrine (closed circles), or $l$-norepinephrine (open triangles) was administered intravenously during the periods marked "Infusion." With one exception (P. D.) in Table I, in whom only periods 8 and 9 were obtained during recovery, all data presented here were collected in complete experiments. Three experiments are thus averaged together for each substance, viz., epinephrine (USP)-H. J., C. N., and G. G.; l-epinephrine-A. B., M. W. and G. I. ; l-norepinephrineI. C., A. B., and E. R. (See Table I for further information regarding these subjects.) The urine flow rose slightly during the infusion of all the pressor amines and fell sharply on withdrawal during the "Recovery" periods.

subjective responses to epinephrine (USP), $l$-epinephrine and $l$-norepinephrine observed during the course of this study were similar to those reported previously (13-15). Epinephrine (USP) and $l$-epinephrine had a similar effect in producing an elevation in systolic pressure with slight or no change in diastolic pressure. On the average, systolic pressure increased 20 and $35 \mathrm{~mm}$. $\mathrm{Hg}$ and diastolic increased 4 and $8 \mathrm{~mm}$. $\mathrm{Hg}$, respectively, in association with tachycardia. Despite these relatively slight changes in arterial tension, the subjects usually complained of nervousness and palpitation, the heart rate was occasionally irregular (premature contractions) and tremor and pallor were often striking. On termination of the infusion the skin became flushed and a sensation of increased body warmth was experienced. In contrast, $l$-norepinephrine in approximately equal dosage brought about a more striking elevation in both systolic (averaging $50 \mathrm{~mm} . \mathrm{Hg}$ ) and diastolic $(25 \mathrm{~mm}$. $\mathrm{Hg}$ ) pressures in association with a marked bradycardia and very little subjective disturbance. Some subjects complained of slight headache, dizziness, substernal oppression and breathlessness; others suffered no discomfort whatever. Hyperemia of the skin was not noticeable following withdrawal of the drug.

Renal vascular adjustments. Despite these differences in systemic hemodynamic activity all three substances affected renal function similarly. The averaged figures for renal plasma flow (RPF) and glomerular filtration rate (GFR) during the control period, during the infusion of the pressor agent and during the recovery period are presented in Table I. It can be seen that renal plasma flow (RPF) decreased in all but one (H. J.), in whom $l$-epinephrine produced renal vasoconstriction proportionate to the elevation in arterial pressure so that there was no change in RPF. Glomerular filtration (GFR) remained relatively unchanged or increased slightly so that the filtration fraction (GFR/RPF) always rose. The calculated renal vascular resistance was invariably increased-to a much greater extent by $l$-norepinephrine than by either $l$-epinephrine or epinephrine (USP). These changes were rapidly reversible on termination of the infusion. A moderate increase in renal blood flow was observed approximately 30 minutes following epinephrine (USP) and $l$-epinephrine that did not develop following $l$-norepinephrine.

Changes in water and electrolyte output. All three agents influenced the urinary excretion of sodium, potassium, and water in the same way. The plasma sodium and potassium concentration did not change significantly at any time. Sodium and potassium output in the urine usually decreased sharply and significantly ${ }^{8}$ whereas urine

8 Statistical analysis indicates that these changes are highly significant. Although electrolyte output varied widely from subject to subject, the variation within any single individual was relatively small during periods of time equivalent to those covered in these experiments. Similar studies of inactive agents, such as small doses of isotopes, revealed no changes in electrolyte excretion greater than those occurring spontaneously during the control periods. Hence, the observations reported here cannot be accounted for on the basis of chance variations or of nonspecific effects of the experimental procedure. 
flow showed no consistent directional change. On termination of the infusion and on the return of blood pressure to normal values, sodium and potassium excretion tended to return toward control levels and urine flow usually fell off abruptly (Table I and Figures 1 to 3 ). Urine flow (V) increased markedly in two of the three patients receiving epinephrine (USP) and in all three fell off to or below the control level during recovery. The urine flow increased (Table I) significantly in only one of three studies with $l$-epinephrine and decreased sharply during recovery in all three. The diuretic tendency was much less evident during the action of $l$-norepinephrine. Averaged urine flows were observed to rise in three (P. D., T. G., F. E.), remain relatively unchanged in six, and fall in three (D. C., K. N., P. M.). In two of four measurements made during recovery the urine flow diminished. Since many of these patients were responding by diuresis to water ingested prior to the study, the rise in urine flow may have been coincidental. In Figure 1, the values for urine flow during each clearance measurement have been averaged and plotted as the percentage of the total control mean values, using only those experiments in which recovery was followed for at least 30 minutes. The discard period excluded from the averaged re-

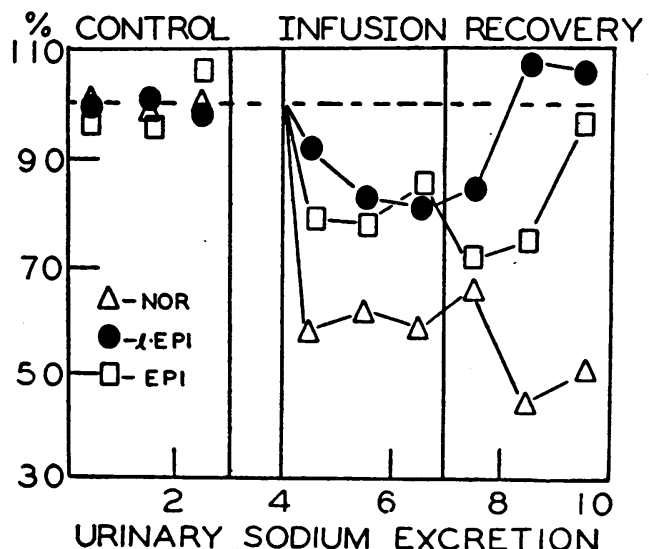

Fig. 2. The Effect of EpINephrine (USP), $l$-EPINEPHRINE, AND $l$-NOREPINEPHRINE ON URINARY SODIUM EXCRETION

The data for urinary sodium excretion in the subjects presented in Figure 1 are handled here in the same manner. Sodium excretion decreased during the drug action and tended to return to the control levels following termination of the infusion.

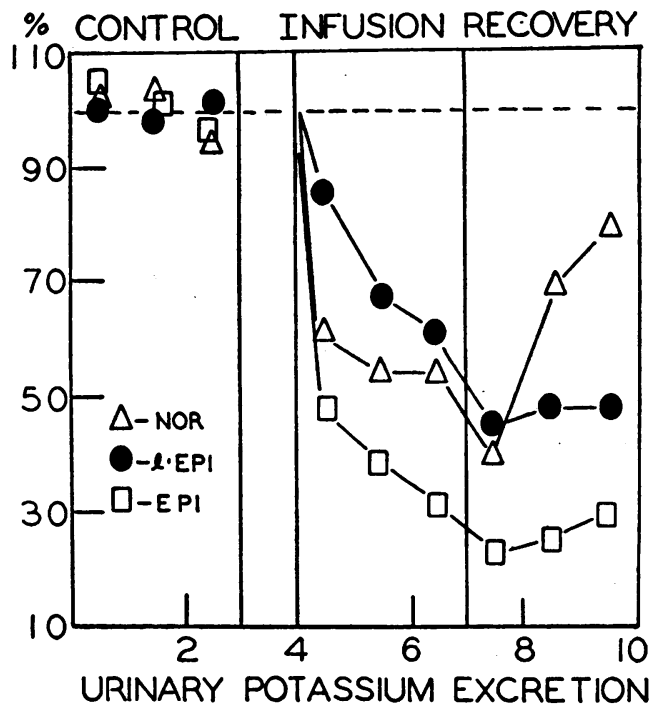

Fig. 3. The Effect of EpINephrine (USP), l-EpiNEPhRINE, AND l-NoRepinephrine on URINARY PotasSIUM EXCRETION

The data are treated as in Figure 1. Potassium excretion decreased markedly during infusion of each drug and tended to remain low thereafter.

covery values in Table $I$ have been included in this figure. The similarity of the response to $l$-norepinephrine, $l$-epinephrine, and epinephrine (USP) is obvious. This figure brings out more clearly than the averages presented in Table I the tendency for urine flow to fall below the control values during recovery from the pressor effect, and indicates the lack of clear-cut evidence for the development of water diuresis during pressor activity.

Sodium excretion (Una V in Table I) decreased on the average during the infusion of epinephrine (USP) and $l$-epinephrine in two or three studies each, and in nine of 11 during infusion of $l$-norepinephrine. During the recovery period sodium excretion returned to or above the control in one subject after epinephrine (USP) and in two after $l$-epinephrine. It did not reach the control level in any of the three followed during recovery from the action of $l$-norepinephrine. These effects are more obvious in Figure 2 which is constructed like Figure 1, on the basis of averaged values for each clearance period, including the recovery discard period expressed in terms of the total control mean. It can be seen that $l$-norepinephrine appeared to have a much 
more profound effect in depressing sodium excretion than either $l$-epinephrine or epinephrine (USP). As a rule sodium excretion fell off much more than urine flow and the urinary concentration of sodium diminished (Table I). Sodium excretion decreased more than the filtration rate so that the sodium-inulin clearance ratio ( $\mathrm{Cna} / \mathrm{Cin}$ in Table I) fell in all but P. M. and E. B. ( $l$-norepinephrine), indicating augmented tubular reabsorption of sodium.

Potassium excretion was also depressed by the action of epinephrine (USP), $l$-epinephrine, and $l$-norepinephrine. This change occurred without exception and persisted throughout the recovery period (Table I and Figure 3 ). The urinary potassium concentration always decreased and the potassium-inulin clearance ratio $(\mathrm{Ck} / \mathrm{Cin}$ in Table I) fell and remained low following recovery. Hence, the tubular reabsorption of potassium was always augmented by the adrenal medullary hormones. In general, $l$-norepinephrine appeared to produce a more profound change in sodium excretion than in potassium excretion whereas l-epinephrine had a greater effect on the output of potassium.

\section{DISCUSSION}

Despite a noteworthy divergence in systemic hemodynamic activity, $l$-norepinephrine and $l$-epinephrine produce more or less identical changes in renal function. Epinephrine (USP), a mixture of the two, behaves like $l$-epinephrine in its action upon the circulation and the kidney. Overall vasodilatation is manifest in elevated cardiac output with little or no change in mean arterial pressure during the action of $l$-epinephrine and epinephrine (USP), whereas generalized vasoconstriction is evident in diminished cardiac output and elevated arterial pressure during the response to $l$-norepinephrine $(3,14,15)$. All excite a renal vasoconstriction that reduces renal blood flow without significant change in filtration (1-3). The present study has revealed further that all appear to interfere to some extent with tubular reabsorption of water and that all give rise to augmented tubular reabsorption of sodium

TABLE I

The effect of adrenal medullary hormones on renal function *

\begin{tabular}{|c|c|c|c|c|c|c|c|c|c|c|c|c|c|}
\hline Sex & $\begin{array}{l}\text { Subject } \\
\text { Age }\end{array}$ & $\stackrel{\text { S.A. }}{M^{2}}$ & Procedure & $\begin{array}{c}\text { B.P. } \\
m m . \mathrm{Hg}\end{array}$ & $\begin{array}{c}\text { P.R. } \\
\text { per } \\
\text { min. }\end{array}$ & $\begin{array}{c}\mathrm{V} \\
\operatorname{ml} . / \min .\end{array}$ & $\begin{array}{c}\text { GFR } \\
m l . / m i n .\end{array}$ & $\begin{array}{c}\mathrm{RPF} \\
\operatorname{ml} . / \min .\end{array}$ & $\begin{array}{l}\text { FF } \\
\%\end{array}$ & $\begin{array}{c}\text { Una V } \\
\mu E q . / \min .\end{array}$ & $\begin{array}{c}\mathrm{Uk} \mathrm{V} \\
\mu E q . / \min .\end{array}$ & $\begin{array}{c}\text { Cna/Cin } \\
\%\end{array}$ & $\begin{array}{c}\mathrm{Ck} / \mathrm{Cin} \\
\%\end{array}$ \\
\hline \multicolumn{14}{|c|}{ epinephrine (USP) } \\
\hline $\mathbf{M}$ & $\begin{array}{l}\text { H. J. } \\
49\end{array}$ & 1.55 & $\begin{array}{l}\text { Control } \\
\text { Infusion } \\
\text { Recovery }\end{array}$ & $\begin{array}{l}118 / 80 \\
138 / 80 \\
122 / 72\end{array}$ & $\begin{array}{l}72 \\
80 \\
74\end{array}$ & $\begin{array}{l}1.6 \\
6.1 \\
1.1\end{array}$ & $\begin{array}{r}90 \\
95 \\
110\end{array}$ & $\begin{array}{l}470 \\
480 \\
555\end{array}$ & $\begin{array}{r}19 \\
20 \\
\cdot 20\end{array}$ & $\begin{array}{l}74.6 \\
55.2 \\
89.2\end{array}$ & $\begin{array}{l}45.3 \\
21.0 \\
20.6\end{array}$ & $\begin{array}{l}0.59 \\
0.42 \\
0.56\end{array}$ & $\begin{array}{r}12.96 \\
5.65 \\
4.72\end{array}$ \\
\hline $\mathbf{F}$ & $\underset{37}{\text { C. N. }}$ & 1.67 & $\begin{array}{l}\text { Control } \\
\text { Infusion } \\
\text { Recovery }\end{array}$ & $\begin{array}{r}100 / 64 \\
130 / 70 \\
98 / 58\end{array}$ & $\begin{array}{l}54 \\
75 \\
60\end{array}$ & $\begin{array}{l}6.3 \\
8.0 \\
0.8\end{array}$ & $\begin{array}{r}105 \\
105 \\
95\end{array}$ & $\begin{array}{l}500 \\
375 \\
520\end{array}$ & $\begin{array}{l}21 \\
28 \\
18\end{array}$ & $\begin{array}{r}103.1 \\
97.6 \\
92.1\end{array}$ & $\begin{array}{l}63.3 \\
18.3 \\
11.7\end{array}$ & $\begin{array}{l}0.72 \\
0.68 \\
0.72\end{array}$ & $\begin{array}{r}13.74 \\
3.94 \\
2.83\end{array}$ \\
\hline $\mathbf{M}$ & $\begin{array}{l}\text { G. G. } \\
39\end{array}$ & 1.95 & $\begin{array}{l}\text { Control } \\
\text { Infusion } \\
\text { Recovery }\end{array}$ & $\begin{array}{l}120 / 70 \\
138 / 74 \\
124 / 64\end{array}$ & $\begin{array}{l}70 \\
85 \\
74\end{array}$ & $\begin{array}{r}12.2 \\
13.3 \\
1.5\end{array}$ & $\begin{array}{l}130 \\
145 \\
130\end{array}$ & $\begin{array}{l}680 \\
550 \\
740\end{array}$ & $\begin{array}{l}19 \\
27 \\
18\end{array}$ & $\begin{array}{l}373.6 \\
277.8 \\
202.2\end{array}$ & $\begin{array}{r}145.4 \\
65.6 \\
27.9\end{array}$ & $\begin{array}{l}2.04 \\
1.37 \\
1.14\end{array}$ & $\begin{array}{r}24.28 \\
10.10 \\
4.74\end{array}$ \\
\hline \multicolumn{14}{|c|}{ lepinephrine } \\
\hline $\mathbf{M}$ & $\underset{44}{\text { A. B. }}$ & 1.64 & $\begin{array}{l}\text { Control } \\
\text { Infusion } \\
\text { Recovery }\end{array}$ & $\begin{array}{l}120 / 84 \\
148 / 84 \\
128 / 84\end{array}$ & $\begin{array}{r}95 \\
110 \\
105\end{array}$ & $\begin{array}{l}0.9 \\
0.8 \\
1.0\end{array}$ & $\begin{array}{l}105 \\
105 \\
115\end{array}$ & $\begin{array}{l}440 \\
395 \\
455\end{array}$ & $\begin{array}{l}24 \\
27 \\
25\end{array}$ & $\begin{array}{r}86.5 \\
81.0 \\
118.0\end{array}$ & $\begin{array}{l}50.8 \\
33.1 \\
23.6\end{array}$ & $\begin{array}{l}0.63 \\
0.59 \\
0.79\end{array}$ & $\begin{array}{r}10.00 \\
7.19 \\
4.27\end{array}$ \\
\hline $\mathbf{F}$ & $\underset{26}{\text { M. W. }}$ & 1.58 & $\begin{array}{l}\text { Control } \\
\text { Infusion } \\
\text { Recovery }\end{array}$ & $\begin{array}{l}100 / 74 \\
140 / 82 \\
100 / 62\end{array}$ & $\begin{array}{l}76 \\
90 \\
90\end{array}$ & $\begin{array}{l}5.5 \\
9.1 \\
1.8\end{array}$ & $\begin{array}{r}110 \\
95 \\
115\end{array}$ & $\begin{array}{l}540 \\
340 \\
560\end{array}$ & $\begin{array}{l}21 \\
28 \\
20\end{array}$ & $\begin{array}{r}118.4 \\
90.5 \\
123.0\end{array}$ & $\begin{array}{l}21.4 \\
16.1 \\
14.4\end{array}$ & $\begin{array}{l}0.81 \\
0.71 \\
0.83\end{array}$ & $\begin{array}{l}5.23 \\
4.50 \\
3.40\end{array}$ \\
\hline F & $\underset{29}{\text { G. I. }}$ & 1.53 & $\begin{array}{l}\text { Control } \\
\text { Infusion* } \\
\text { Recovery }\end{array}$ & $\begin{array}{l}114 / 76 \\
150 / 90 \\
118 / 50\end{array}$ & $\begin{array}{l}72 \\
94 \\
74\end{array}$ & $\begin{array}{l}7.8 \\
6.2 \\
2.8\end{array}$ & $\begin{array}{l}100 \\
115 \\
125\end{array}$ & $\begin{array}{l}600 \\
495 \\
745\end{array}$ & $\begin{array}{l}17 \\
24 \\
17\end{array}$ & $\begin{array}{l}78.6 \\
63.8 \\
61.4\end{array}$ & $\begin{array}{l}85.3 \\
57.3 \\
26.2\end{array}$ & $\begin{array}{l}0.58 \\
0.41 \\
0.36\end{array}$ & $\begin{array}{r}19.70 \\
11.36 \\
4.87\end{array}$ \\
\hline
\end{tabular}


TABLE I-Continued

\begin{tabular}{|c|c|c|c|c|c|c|c|c|c|c|c|c|c|}
\hline Sex & $\begin{array}{l}\text { Subject } \\
\text { Age }\end{array}$ & S.A. $_{\mathbf{M}^{\mathbf{2}}}$ & Procedure & $\begin{array}{c}\text { B.P. } \\
m m . H g\end{array}$ & $\begin{array}{l}\text { P.R. } \\
\text { per } \\
\text { min. }\end{array}$ & $\begin{array}{c}\mathrm{V} \\
\text { ml./min. }\end{array}$ & $\begin{array}{c}\text { GFR } \\
\text { ml./min. }\end{array}$ & $\begin{array}{c}\text { RPF } \\
\text { ml./min. }\end{array}$ & $\begin{array}{l}\text { FF } \\
\%\end{array}$ & $\begin{array}{c}\text { Una V } \\
\mu E q . / m i n .\end{array}$ & $\begin{array}{c}\mathrm{Uk} \mathrm{V} \\
\mu E q . / m i n .\end{array}$ & $\begin{array}{c}\text { Cna/Cin } \\
\%\end{array}$ & $\begin{array}{c}\mathrm{Ck} / \mathrm{Cin} \\
\%\end{array}$ \\
\hline \multicolumn{14}{|c|}{$l$-norepinephrine } \\
\hline $\mathbf{F}$ & I. C. & 1.56 & $\begin{array}{l}\text { Control } \\
\text { Infusion } \\
\text { Recovery }\end{array}$ & $\begin{array}{l}100 / 62 \\
150 / 90 \\
106 / 60\end{array}$ & $\begin{array}{l}70 \\
56 \\
70\end{array}$ & $\begin{array}{l}7.9 \\
8.9 \\
0.8\end{array}$ & $\begin{array}{l}145 \\
155 \\
155\end{array}$ & $\begin{array}{l}665 \\
465 \\
550\end{array}$ & $\begin{array}{l}22 \\
33 \\
26\end{array}$ & $\begin{array}{r}176.4 \\
54.0 \\
74.4\end{array}$ & & $\begin{array}{l}0.87 \\
0.25 \\
0.34\end{array}$ & $\begin{array}{l}8.04 \\
5.00 \\
5.15\end{array}$ \\
\hline $\mathbf{M}$ & ${ }_{44}^{\text {A. B. }}$ & 1.64 & $\begin{array}{l}\text { Control } \\
\text { Infusion } \\
\text { Recovery }\end{array}$ & $\begin{array}{l}124 / 88 \\
172 / 108 \\
124 / 90\end{array}$ & $\begin{array}{r}94 \\
90 \\
105\end{array}$ & $\begin{array}{l}1.3 \\
1.2 \\
2.5\end{array}$ & $\begin{array}{r}105 \\
75 \\
105\end{array}$ & $\begin{array}{l}425 \\
270 \\
440\end{array}$ & $\begin{array}{l}24 \\
28 \\
24\end{array}$ & $\begin{array}{r}104.3 \\
28.3 \\
61.6\end{array}$ & & $\begin{array}{l}0.75 \\
0.29 \\
0.44\end{array}$ & $\begin{array}{r}12.03 \\
7.21 \\
9.34\end{array}$ \\
\hline F & ${ }_{22}^{E .} R$. & 1.32 & $\begin{array}{l}\text { Control } \\
\text { Infusion } \\
\text { Recovery* }\end{array}$ & $\begin{array}{r}88 / 54 \\
132 / 94 \\
98 / 56\end{array}$ & $\begin{array}{r}104 \\
75 \\
74\end{array}$ & $\begin{array}{l}2.7 \\
2.0 \\
2.4\end{array}$ & $\begin{array}{l}85 \\
55 \\
85\end{array}$ & & $\begin{array}{l}25 \\
44 \\
23\end{array}$ & $\begin{array}{l}40.1 \\
11.4 \\
26.4\end{array}$ & & $\begin{array}{l}0.37 \\
0.15 \\
0.24\end{array}$ & $\begin{array}{r}15.22 \\
8.33 \\
13.45\end{array}$ \\
\hline $\mathbf{M}$ & $\mathrm{P}_{47} \mathrm{D}$ & 1.89 & $\begin{array}{l}\text { Control } \\
\text { Infusion } \\
\text { Recovery }\end{array}$ & $\begin{array}{l}120 / 80 \\
170 / 102 \\
110 / 74\end{array}$ & $\frac{66}{68}$ & $\begin{array}{r}7.4 \\
12.7 \\
2.8\end{array}$ & $\begin{array}{r}140 \\
135 \\
95\end{array}$ & $\begin{array}{l}710 \\
560 \\
500\end{array}$ & $\begin{array}{l}20 \\
24 \\
19\end{array}$ & 三 & 三 & = & $\bar{z}$ \\
\hline $\mathbf{M}$ & ${ }_{30}^{\text {L. F. }}$ & 1.93 & $\begin{array}{l}\text { Control } \\
\text { Infusion }\end{array}$ & $\begin{array}{l}108 / 80 \\
158 / 106\end{array}$ & $\begin{array}{l}64 \\
60\end{array}$ & $\begin{array}{l}7.1 \\
6.3\end{array}$ & $\begin{array}{r}90 \\
120\end{array}$ & $\begin{array}{l}520 \\
485\end{array}$ & $\begin{array}{l}18 \\
24\end{array}$ & $\begin{array}{l}96.7 \\
47.1\end{array}$ & $\begin{array}{l}92.9 \\
78.2\end{array}$ & $\begin{array}{l}0.72 \\
0.29\end{array}$ & $\begin{array}{l}25.30 \\
14.60\end{array}$ \\
\hline $\mathbf{M}$ & $\underset{52}{H . P .}$ & 1.74 & $\begin{array}{l}\text { Control } \\
\text { Infusion }\end{array}$ & \begin{tabular}{l|}
$112 / 70$ \\
$164 / 90$
\end{tabular} & $\begin{array}{l}75 \\
50\end{array}$ & $\begin{array}{l}9.0 \\
9.3\end{array}$ & $\begin{array}{l}85 \\
90\end{array}$ & $\begin{array}{l}390 \\
315\end{array}$ & $\begin{array}{l}22 \\
29\end{array}$ & $\begin{array}{l}161.2 \\
140.9\end{array}$ & $\begin{array}{l}55.2 \\
36.2\end{array}$ & $\begin{array}{l}1.38 \\
1.12\end{array}$ & $\begin{array}{r}13.20 \\
8.03\end{array}$ \\
\hline $\mathbf{F}$ & $\underset{18}{\text { D. C. }}$ & 1.55 & $\begin{array}{l}\text { Control } \\
\text { Infusion }\end{array}$ & $\begin{array}{c}94 / 54 \\
166 / 100\end{array}$ & $\begin{array}{l}72 \\
60\end{array}$ & $\begin{array}{l}5.8 \\
4.1\end{array}$ & $\begin{array}{l}90 \\
95\end{array}$ & $\begin{array}{l}535 \\
295\end{array}$ & $\begin{array}{l}17 \\
32\end{array}$ & $\begin{array}{r}118.1 \\
67.2\end{array}$ & $\begin{array}{l}63.2 \\
23.3\end{array}$ & $\begin{array}{l}0.91 \\
0.50\end{array}$ & $\begin{array}{r}18.10 \\
6.33\end{array}$ \\
\hline $\mathbf{M}$ & $\mathrm{T}_{50} \mathrm{G}$. & 1.85 & $\begin{array}{l}\text { Control } \\
\text { Infusion }\end{array}$ & $\begin{array}{l}110 / 72 \\
168 / 90\end{array}$ & $\begin{array}{l}60 \\
52\end{array}$ & $\begin{array}{r}2.4 \\
10.5\end{array}$ & $\begin{array}{l}115 \\
110\end{array}$ & $\begin{array}{l}650 \\
435\end{array}$ & $\begin{array}{l}19 \\
25\end{array}$ & $\begin{array}{l}115.1 \\
104.0\end{array}$ & $\begin{array}{r}104.0 \\
77.3\end{array}$ & $\begin{array}{l}0.77 \\
0.52\end{array}$ & $\begin{array}{l}21.19 \\
16.80\end{array}$ \\
\hline $\mathbf{F}$ & $\mathrm{K}_{\mathbf{3 0}} \mathbf{N}$. & 1.46 & $\begin{array}{l}\text { Control } \\
\text { Infusion }\end{array}$ & $\begin{array}{l}122 / 62 \\
146 / 82\end{array}$ & $\begin{array}{l}96 \\
68\end{array}$ & $\begin{array}{r}13.2 \\
8.4\end{array}$ & $\begin{array}{r}105 \\
90\end{array}$ & $\begin{array}{l}540 \\
325\end{array}$ & $\begin{array}{l}19 \\
28\end{array}$ & $\begin{array}{l}93.6 \\
40.2\end{array}$ & $\begin{array}{l}52.8 \\
32.7\end{array}$ & $\begin{array}{l}0.65 \\
0.31\end{array}$ & $\begin{array}{r}12.78 \\
9.29\end{array}$ \\
\hline $\mathbf{F}$ & $\underset{34}{F . E .}$ & 1.90 & $\begin{array}{l}\text { Control } \\
\text { Infusion }\end{array}$ & $\begin{array}{l}112 / 74 \\
146 / 88\end{array}$ & $\begin{array}{l}85 \\
78\end{array}$ & $\begin{array}{l}6.2 \\
8.5\end{array}$ & $\begin{array}{l}115 \\
110\end{array}$ & $\begin{array}{l}575 \\
430\end{array}$ & $\begin{array}{l}20 \\
25\end{array}$ & $\begin{array}{r}101.0 \\
80.0\end{array}$ & $\begin{array}{l}81.2 \\
49.1\end{array}$ & $\begin{array}{l}0.64 \\
0.54\end{array}$ & $\begin{array}{l}17.86 \\
11.22\end{array}$ \\
\hline $\mathbf{F}$ & ${ }_{28}^{\text {P. M. }}$ & 1.87 & $\begin{array}{l}\text { Control } \\
\text { Infusion }\end{array}$ & $\begin{array}{r}94 / 60 \\
144 / 88\end{array}$ & $\begin{array}{l}65 \\
55\end{array}$ & $\begin{array}{r}11.9 \\
9.7\end{array}$ & $\begin{array}{l}105 \\
105\end{array}$ & $\begin{array}{l}630 \\
500\end{array}$ & $\begin{array}{l}17 \\
22\end{array}$ & $\begin{array}{l}164.2 \\
169.3\end{array}$ & $\begin{array}{r}130.0 \\
83.6\end{array}$ & $\begin{array}{l}1.11 \\
1.27\end{array}$ & $\begin{array}{l}27.20 \\
17.20\end{array}$ \\
\hline $\mathbf{F}$ & $\underset{25}{\mathrm{E} .} \mathrm{B}$. & 1.72 & $\begin{array}{l}\text { Control } \\
\text { Infusion }\end{array}$ & $\begin{array}{l}102 / 66 \\
138 / 92\end{array}$ & $\begin{array}{l}80 \\
65\end{array}$ & $\begin{array}{l}8.6 \\
8.8\end{array}$ & $\begin{array}{l}130 \\
135\end{array}$ & $\begin{array}{l}535 \\
495\end{array}$ & $\begin{array}{l}24 \\
28\end{array}$ & $\begin{array}{l}51.5 \\
73.4\end{array}$ & $\begin{array}{l}37.3 \\
29.5\end{array}$ & $\begin{array}{l}0.30 \\
0.40\end{array}$ & $\begin{array}{l}6.01 \\
4.47\end{array}$ \\
\hline
\end{tabular}

* All values are averages of two or more determinations except in instances marked with an asterisk. Infusions of epinephrine (USP), l-epinephrine, or l-norepinephrine were administered throughout "infusion" period at a rate sufficient to produce an elevation in arterial pressure without undue discomfort. The arterial pressure (B.P.) was measured sphygmomanometrically. Abbreviations are as follows:

S. A. = body surface $\left(M^{2}\right)$

P. R. = pulse rate

$\mathrm{V}=$ urine flow (ml./min.)

GFR = glomerular filtration rate, inulin clearance ( $\mathrm{ml} . / \mathrm{min}$.)

$\mathrm{RPF}=$ renal plasma flow, sodium p-aminohippurate clearance ( $\mathrm{ml} . / \mathrm{min}$.)

FF = filtration fraction-GFR/RPF (\%)

Una $\mathrm{V}=$ urinary sodium output $(\mu \mathrm{Eq} . / \mathrm{min}$.)

Uk V = urinary potassium output ( $\mu \mathrm{Eq} . / \mathrm{min}$.

$\mathrm{Cna} / \mathrm{Cin}=$ sodium-inulin clearance ratio or proportion of filtered sodium excreted (\%)

$\mathrm{Ck} / \mathrm{Cin}=$ potassium-inulin clearance ratio or proportion of filtered potassium excreted $(\%)$

and potassium. Since maximal tubular reabsorptive (glucose $\mathrm{Tm}$ ) and excretory (diodrast $\mathrm{Tm}$ ) capacities are not affected by epinephrine (USP) (16), it is not likely that either $l$-epinephrine or $l$-norepinephrine have any effect on $\mathrm{Tm}$. It may be inferred from this and much additional direct evidence (17-19) that the renal vasculature and parenchyma are diffusely and totally involved in the observed response.

The mechanism of the change in urine formation is not yet apparent. Since it appears when blood pressure is little altered or, contrariwise. 
greatly increased, when tachycardia indicates increased cardiac output during the action of $l$-epinephrine, or when bradycardia denotes diminished output with $l$-norepinephrine, it may be presumed that the general systemic hemodynamic adjustment is not a determining factor. Attention must be paid rather to the local renal circulatory adjustments and to the possibility of direct or indirect influence upon tubular cellular activity by the drugs themselves.

The site of intrarenal vasoconstriction has been placed by Smith and others in the post-glomerular vascular bed. This view is based upon the observations of Richards and Plant $(20,21)$ that perfused kidneys may swell during the action of adrenal extracts (and presumably also $l$-epinephrine), and of Smith and his associates $(1,22)$ that glomerular filtration does not decrease. The first is interpreted as evidence for engorgement of a sizeable portion of the renal vascular bed proximal to the point of constriction. Since filtration does not increase it may be presumed that intraglomerular filtration pressure must rise in proportion to the decrement in renal plasma flow independently of any alteration in systemic arterial pressure. In this view afferent arteriolar constriction develops in proportion to the elevation in the blood pressure and vasoconstriction in the post-glomerular vascular bed accounts for depression of renal blood flow below the control value. Gomez $(22,23)$ has placed the site of this activity in the venular bed in order to account for the swelling of perfused kidneys. From this it follows that intrarenal pressure may rise and bring about the observed augmentation in tubular reabsorption of sodium. But studies (24) of the effect of increased intra-abdominal pressure, that presumably increases intrarenal tension, indicate that water reabsorption is also increased and that potassium reabsorption is relatively unaffected. Hence, it seems unlikely that increased interstitial pressure in the kidney was an important determining factor in these studies.

The adrenal medulla is part and parcel of a widespread integrated regulatory system that includes the autonomic nervous system and, among other organs, the adrenal cortex and the pituitary. Although the renal excretory response to medullary hormones may be mediated in part by neural pathways, the temporal relationships between' stimulus, response, and recovery suggest that humoral mechanisms are operative. Some humoral agents such as antidiuretic hormone and desoxycorticosterone acetate appear to affect renal excretion of water and electrolytes by direct action upon the renal tubules. It is possible that the adrenal medullary hormones behave in this manner or that they alter the production of active agents by other organs. Secondary release of adrenal cortical hormones or adrenocorticotrophic hormone (25) might thus account for the increment in tubular reabsorption of sodium, although these substances would be expected to promoteand not depress-potassium excretion (26). The marked fall in urine flow on withdrawal of these agents suggests that they may interfere with tubular reabsorption of water, an effect which may be attributable to diminished secretion of antidiuretic hormone (27). Perhaps the electrolyte-retaining effect of adrenal medullary hormones is attributable, in part at least, to an altered cellular metabolism. The resultant shifts in electrolyte and water distribution may ultimately influence renal function directly through derangements in the composition of the blood. The relative importance of these factors is under investigation.

Presumably any condition or situation that stimulates release of large amounts of epinephrine and norepinephrine is one in which renal retention of sodium and potassium occurs. However, pheochromocytomata are not reported to have this effect. Hence it is probable that the changes observed in the course of this study occur only as an immediate response and that continued change is prevented by corrective adjustments to restore electrolyte output to normal. It is unlikely, therefore, that epinephrine and norepinephrine play a role in producing the defect in sodium excretion enco'intered in such conditions as cardiac decompensation. On the other hand, the decrease in electrolyte excretion during anesthesia (28) and exercise (29), for example, may be ascribed to the action of adrenal medullary hormones upon the tubules as well as to diminished glomerular filtration.?

\footnotetext{
- Since this paper was submitted for publication several papers dealing with this subject have appeared. Jacobson, Hammarsten, and Heller (30) have confirmed in general the observations reported here. They found that epinephrine (USP) caused a reduction in sodium and
} 


\section{SUM MARY}

Epinephrine (USP), $l$-epinephrine, and $l$-norepinephrine have been found to depress the urinary excretion of sodium and potassium in normal human subjects. Since the glomerular filtration rate remained relatively constant despite elevated arterial pressure, intrarenal vasoconstriction, and renal ischemia, the change in electrolyte output must be ascribed to augmented tubular reabsorption. Urine flow usually increased somewhat during the action of the adrenal medullary hormones and fell off sharply on withdrawal, suggesting that these drugs interfere with tubular reabsorption of water.

\section{ACKNOWLEDGMENTS}

We are deeply indebted to Miss Lottie Fogel, Miss Joan Hunt, and Mrs. Michaeleen Patterson for technical assistance.

We wish to thank Dr. John Fertig for assistance in the statistical analysis.

\section{REFERENCES}

1. Chasis, H., Ranges, H. A., Goldring, W., and Smith, H. W., The control of renal blood flow and glomerular filtration in normal man. J. Clin. Invest., 1938, 17, 683.

2. Barnett, A. J., Blacket, R. B., Depoorter, A. E., Sanderson, P. H., and Wilson, G. M., The action of noradrenalin in man and its relation to phaeochromocytoma and hypertension. Clin. Sc., 1950, 9, 151.

3. Ranges, H. A., and Bradley, S. E., Systemic and renal circulatory changes following the administration of adrenin, ephedrine and paredrinol to normal man. J. Clin. Invest., 1943, 22, 687.

4. Stein, L., and Wertheimer, E., Disturbances of water balance in the rat on removal of the adrenal medulla. J. Endocrinol., 1944, 3, 356.

5. Gaunt, R., Liling, M., and Cordsen, M., Adrenal medulla in water diuresis and water intoxication. Endocrinology, 1945, 37, 136.

potassium excretion. Unfortunately the doses of epinephrine employed produced changes in plasma potassium and glomerular filtration rate that made interpretation impossible. These effects were avoided in the course of the study reported here. Two other papers $(31,32)$ deal with electrolyte excretion in a small group of subjects given epinephrine (USP) over a period of three days. Sodium output increased whereas potassium output diminished. This phenomenon is not germane to the present discussion but it lends further support to the view expressed above that the acute response is probably of relatively short duration.
6. Hughes-Jones, N. C., Pickering, G. W., Sanderson, P. H., Scarborough, H., and Vandenbroucke, J., The nature of the action of renin and hypertension on renal function in the rabbit. J. Physiol., 1949, 109, 288.

7. Horres, A. D., Eversole, W. J., and Rock, M., Adrenal medullary hormones in water diuresis. Proc. Soc. Exper. Biol. \& Med., 1950, 75, 58.

8. Blake, W. D., Effect of adrenaline on renal excretion of water and sodium. Federation Proc., 1951, 10, 15.

9. Goldenberg, M., Faber, M., Alston, E. J., and Chargaff, E. C., Evidence for the occurrence of norepinephrine in the adrenal medulla. Science, 1949, 109, 534.

10. Tullar, B. F., The separation of $l$-arterenol from natural U.S.P. epinephrine. Science, 1949, 109, 536.

11. Böger, A., and Wezler, K., Die Bestimmung des arteriellen Gesamtwiderstandes am Menschen. Arch. f. exper. Path. u. Pharmakol., 1937, 186, 43.

12. Goldring, W., and Chasis, H., Hypertension and Hypertensive Disease. The Commonwealth Fund, New York, 1944, pp. 195-207.

13. Bradley, S. E., Bradley, G. P., Tyson, C. J., Curry, J. J., and Blake, W. D., Renal function in renal diseases. Am. J. Med., 1950, 9, 766.

14. Goldenberg, M., Pines, K. L., Baldwin, E. deF., Greene, D. G., and Roh, C. E., The hemodynamic response of man to nor-epinephrine and epinephrine and its relation to the problem of hypertension. Am. J. Med., 1948, 5, 792.

15. Barcroft, H., and Konzett, $H$., On the actions of noradrenaline, adrenaline and isopropyl noradrenaline on the arterial blood pressure, heart rate and muscle blood flow in man. J. Physiol., 1949, 110, 194.

16. Smith, H. W., Goldring, W., Chasis, H., Ranges, H. A., and Bradley, S. E., The application of saturation methods to the study of glomerular and tubular function in the human kidney. J. Mt. Sinai Hosp., 1943, 10, 59.

17. Reubi, F. C., and Schroeder, H. A., Can vascular shunting be induced in the kidney by vasoactive drugs? J. Clin. Invest., 1949, 28, 114.

18. Kahn, J. R., Skeggs, L. T., and Shumway, N. P., Studies of the renal circulation. Circulation, 1950, $1,445$.

19. Houck, C. R., Alterations in renal hemodynamics and function during the intravenous injection of epinephrine in the dog. Am. J. Physiol., 1951, 166, 649.

20. Richards, A. N., and Plant, O. H., Urine formation in the perfused kidney. The influence of adrenalin on the volume of the perfused kidney. Am. J. Physiol., 1922, 59, 184.

21. Richards, A. N., and Plant, O. H., The action of minute doses of adrenalin and pituitrin on the kidney. Am. J. Physiol., 1922, 59, 191.

22. Smith, H. W., The Kidney-Structure and Function 
in Health and Disease. Oxford University Press, New York, 1951, p. 424.

23. Gomez, D. M., L'hémodynamique de la circulation rénale. La Rev. Scientif., 1947, 85, 45.

24. Bradley, S. E., Mudge, G. H., Blake, W. D., and Alphonse, $P$., The effect of increased intra-abdominal pressure upon renal excretion of water, sodium and potassium in normal human subjects and in patients with diabetes insipidus. J. Clin. Invest., 1949, 28, 772.

25. Long, C. N. H., The conditions associated with the secretion of the adrenal cortex. Federation Proc., 1947, 6, 461.

26. Perera, G. A., Pines, K. L., Hamilton, H. B., and Vislocky, K., Clinical and metabolic study of 11dehydro-17-hydroxy-corticosterone acetate (Kendall Compound E) in hypertension, Addison's disease and diabetes mellitus. Am. J. Med., 1949, 7, 56.

27. Verney, E. B., Croonian lecture: The antidiuretic hormone and the factors which determine its release. Proc. Roy. Soc., London, 1947, s.B 135, 25.

28. Habif, D. V., Papper, E. M., Fitzpatrick, H. F., Lowrance, P., Smythe, C. McC., and Bradley, S.
E., The renal and hepatic blood flow, glomerular filtration rate, and urinary output of electrolytes during cyclopropane, ether, and thiopental anesthesia, operation, and the immediate postoperative period. Surgery, 1951, 30, 241.

29. Barclay, J. A., Cooke, W. T., Kenney, R. A., and Nutt, M. E., The effects of water diuresis and exercise on the volume and composition of the urine. Am. J. Physiol., 1947, 148, 327.

30. Jacobson, W. E., Hammarsten, J. F., and Heller, B. I., The effects of adrenaline upon renal function and electrolyte excretion. J. Clin. Invest., 1951, 30, 1503.

31. Bliss, E. L., Rubin, S., and Gilbert, T., The effect of adrenalin on adrenal cortical function. J. Clin. Endocrinol., 1951, 11, 46.

32. Duncan, L. E., Jr., Solomon, D. H., Nichols, M. P., and Rosenberg, E., The effect of the chronic administration of adrenal medullary hormones to man on adrenocortical function and the renal excretion of electrolytes. J. Clin. Invest., 1951, 30, 908.

33. International Congress of Clinical Pathology. Brit. Med. J., 1951, 2, 290. 